\title{
PREFACE: THEMATIC ISSUE IN MATHEMATICAL BIOLOGY AND APPLIED EVOLUTIONARY EQUATIONS
}

\author{
NAVEEN K. VAIDYA AND DHRUBA R. ADHIKARI
}

We are pleased to present the thematic issue entitled "Mathematical Biology and Applied Evolutionary Equations" in Mathematics in Applied Sciences and Engineering (MASE). This issue includes some selected articles from invited authors who presented their research work during the Second International Conference on Applications of Mathematics to Nonlinear Sciences (http://anmaweb.org/AMNS-2019/) held on June 27-30, 2019, in Pokhara, Nepal. The primary focus of the AMNS conference series is to provide a platform for discussion about current advancement of interdisciplinary research on the applications of mathematics to nonlinear sciences.

This thematic issue attempts to highlight some of the latest research trends in evolutionary equations and their applications in natural and life sciences. In this regard, the current issue contains a good mixture of research articles that include the development of theories and formulation of computational methods as well as applications of differential equations to various fields, including mathematical biology. The interesting results and open questions presented in this issue may offer opportunities for further development of advanced evolutionary models and advanced methods for their analysis and computations.

We are very much thankful to the contributing authors for sharing their research and the referees for ensuring high quality of this publication. All submissions have gone through a regular peer review process, leading to the acceptance of these ten papers. We thank all reviewers of these submissions for their valuable evaluations and comments. We also thank Dr. Xingu Zou for his help and support during the review process of these articles. Finally, we sincerely thank MASE for publishing this special issue on it.

We look forward to the continuous support from the global mathematical community for the future AMNS conferences.

\footnotetext{
Guest editor, Department of Mathematics and Statistics, San Diego State University, USA.

E-mail address: nvaidya@sdsu.edu

Guest editor, Department of Mathematics, Kennesaw State University, USA.

E-mail address: dadhikar@kennesaw.edu
} 